\title{
Optimal Control of Leukemic Cell Population Dynamics
}

\author{
X. Dupuis * \\ CMAP, Ecole Polytechnique \& Inria Saclay, 91128 Palaiseau Cedex, France
}

\begin{abstract}
We are interested in optimizing the co-administration of two drugs for some acute myeloid leukemias (AML), and we are looking for in vitro protocols as a first step. This issue can be formulated as an optimal control problem. The dynamics of leukemic cell populations in culture is given by age-structured partial differential equations, which can be reduced to a system of delay differential equations, and where the controls represent the action of the drugs. The objective function relies on eigenelements of the uncontrolled model and on general relative entropy, with the idea to maximize the efficiency of the protocols. The constraints take into account the toxicity of the drugs. We present in this paper the modeling aspects, as well as theoretical and numerical results on the optimal control problem that we get.
\end{abstract}

Keywords and phrases: acute myeloid leukemia, optimal control, delay differential equations, population dynamics, general relative entropy, in vitro therapeutic optimization

Mathematics Subject Classification: 49N90, 49K21, 92C50

\section{Introduction}

Acute myeloid leukemias (AML) are cancers of the myeloid lineage of white blood cells. The process of blood production, called hematopoiesis, takes place in the bone marrow, with hematopoietic stem cells (HSC) at its root. HSC have the abilty to self-renew, i.e. to divide without differentiating, and to differentiate towards any lineage of blood cells by dividing into progenitors. These progenitors are committed stem cells which follow a path of diffenrentiation, producing cells which are more and more engaged into one lineage and lose progressively their ability to self-renew. Once they are fully mature and functional, cells of each lineage are released into the bloodstream. The hematopoiesis consists in the regulation of the self-renewal and the differentiation of cell populations [22]. In AML, the differentiation is blocked at some early stage, leading to the accumulation of immature white blood cells, called blasts, of the myeloid lineage. This blockade being associated with a proliferation advantage, the blasts quickly crowd the bone marrow and are eventually released into the bloodstream.

One of the first mathematical model on hematopoiesis was proposed in 1978 by Mackey and focused on the HSC population dynamics [18]. Mackey considered two phases in his model, a resting phase and a proliferating phase, and described the dynamics of the two HSC sub-populations by a system of delay differential equations; these equations can be justified by age-structured partial differential equations.

\footnotetext{
*Corresponding author. E-mail: xavier.dupuis@cmap.polytechnique.fr
} 
To represent the blockade of the differentiation in AML, Adimy et al. considered the dynamics of cell populations of several maturity stages and developped a multi-compartmental model, where each compartment represents a maturity stage and is again divided in two phases [2]. Özbay et al. proceeded with the stabiliy analysis of this delay differential system in [21], and Avila et al. refined the model in [3] by considering more than two phases per compartement and modeling the fast proliferation in AML. Stiehl and Marciniak also proposed a multi-compartmental model on leukemias [25]; they considered healthy and leukemic cell populations, but did not distinguish resting and proliferating phases and thus did not get delays.

The treatment for most of the types of AML is a challenge [24]. Clinicians of the department of hematology at Saint-Antoine hospital in Paris would be interested for some cases in co-administrating two drugs: a cytotoxic (Aracytin), which enhances cell death, and a cytostatic (AC200), which slows down proliferation. A first step is to determine how such a combination should be scheduled in in vitro experiments. To that purpose, biologists of the same hospital have sampled blood from patients with AML, sorted cancer blasts, and carried out leukemic cell cultures. The number of cells, their state in the cell cycle, and their maturity stage have then been daily measured during 5 days, without and with each of the two drugs at different constant concentrations in the culture [4].

In this paper, we idealize these experiments and consider leukemic cell cultures with varying concentration of both drugs. We are looking for in vitro protocols of drugs administration, i.e. schedules of the concentration of both drugs during the experiment, which are as efficient as possible without being too toxic. To formulate this issue as an optimal control problem, a state equation, an objective function, and constraints have to been set.

The state equation models the cell population dynamics under the action of the drugs; we consider an age-structured model with one maturity compartement, divided in one resting phase and one proliferating phase. Adimy and Crauste used such a model in [1] to represent the dependence of cell death and proliferation on growth factors. Here, the action of the cytotoxic on cell death is age-dependent, and the drug concentrations are not solutions of evolution equations but are control variables which define an in vitro protocol. Gabriel et al. identified the action of a drug inducing quiescence (erlotinib) with a fraction of quiescent cells in [11]. The action of the cytostatic in our model is also represented by a fraction of resting cells, which is here time-dependent, and not by a varying velocity in the proliferating phase as Hinow et al. in [15]. See also [6] about the modeling of the action of the drugs.

The objective function aims at minimizing the leukemic cell population at the end of the experiment, in order to maximize the efficiency of the corresponding protocol. Its definition actually requires a long time asymptotic analysis to avoid an horizon effect. This analysis relies on the specialization of the general relative entropy principle introduced by Michel et al. [20] to our model. Various kinds of objective functions exist in the litterature: final or maximal number of tumor cells [5], final tumor volume [16], performance index [17], or eigenvalue [7]; the use of an age-dependent weight given by eigenelements in this paper seems to be new.

The constraints come from biological bounds on the action of the drugs and from maximal cumulative doses that we impose to limit the toxicity of the protocols, as in [16]; there is no healthy population in our model on which we could set a toxicity threshold as in $[5,7]$. The optimization problem that we get is equivalent, by the method of characteristics, to an optimal control problem of delay differential equations. For such a problem, optimality conditions are available in the form of Pontryagin's minimum principle [14]; it can also be reduced to an undelayed optimal control problem [12,13], and then solved numerically by standard solvers.

The paper is organized as follows. In Section 2, we model the population dynamics under the action of the drugs. Section 3 contains the analysis of this model, including a general relative entropy principle and a long time asymptotic analysis. The optimal control problem is set in Section 4, and theoretical results and numerical optimal protocols are presented in Section 5. The precise statement of Pontryagin's 
minimum principle for our problem has been postponed to the appendix, together with the parameters used for the numerical resolutions.

\section{Modeling}

We present here the dynamics of leukemic cell populations in culture and under the action of the two drugs.

\subsection{Cell Populations}

We consider a leukemic cell population, in vitro, and we distinguish two sub-populations $[1,18]$ : the resting cells, which are inactive $\left(G_{0}\right.$ phase), and the proliferating cells, which are engaged in their cycle $\left(G_{1} S G_{2} M\right.$ phase).

Resting cells are introduced into the proliferating phase at a rate $\beta$, independently of the time spent in the resting phase. Considering that the proliferation is uncontrolled in case of AML, we do not represent any feedback from a cell population $[18,19]$ or a growth factor [1], and thus $\beta$ is constant in our model.

Proliferating cells die by apoptosis at a rate $\gamma$, and if it does not die, a cell divides during mitosis, after a time $2 \tau$ spent in the phase, in two daughter cells which enter the resting phase. We consider that the duration of the proliferating phase $2 \tau$ is the same for all cells; this is not true biologically [2] but one can think of $2 \tau$ as an average duration [19].

We structure the proliferating population by an age variable $a$ which represents the time spent in the proliferating phase by a cell. We denote by $R(t)$ the resting population at time $t$, and by $p(t, a)$ the proliferating population density with age $a$ at time $t$.

\subsection{Action of the Drugs}

The two drugs are a cytotoxic (Aracytin) and a cytostatic (AC220).

The cytotoxic damages the DNA of the cells during the $S$ sub-phase of their cycle; it results in an extra death rate. To simplify further calculus and numerical issues, we consider that $u(t)$, the death rate due to the cytotoxic at time $t$, affects the second-half of the proliferating phase, i.e. proliferating cells with age $a \in[\tau, 2 \tau]$.

The cytostatic inhibits a receptor tyrosine kynase (Flt3) of the cells in the resting phase; it results in a fraction $k(t)$ of inhibited cells among the resting cells, which can no more enter the proliferating phase. The global introduction rate to the proliferating phase at time $t$ is then $(1-k(t)) \beta$.

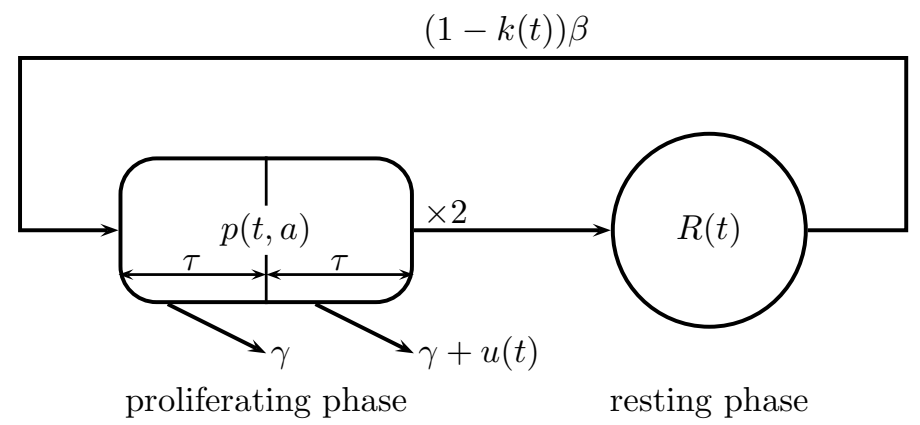

Figure 1 . The model. 
We denote by $v(t)$ the inhibition rate due to the cytostatic at time $t$, and by $\alpha$ the rate of natural dis-inhibition. We consider that the dynamics of $k$ is given by

$$
\frac{\mathrm{d} k}{\mathrm{~d} t}(t)=v(t)(1-k(t))-\alpha k(t) .
$$

The action rates due to the drugs are increasing functions of their concentration in the cell culture, the latter being chosen during in vitro experiments. Thus we consider that we control directly the action rates $u$ and $v$.

\subsection{The Age-Structured Model}

The dynamics of the cell populations is given by the following partially age-structured system:

$$
\begin{array}{ll}
\frac{\mathrm{d} R}{\mathrm{~d} t}(t)=-(1-k(t)) \beta R(t)+2 p(t, 2 \tau) & \\
\frac{\partial p}{\partial t}(t, a)+\frac{\partial p}{\partial a}(t, a)=-\left(\gamma+\chi_{(\tau, 2 \tau)}(a) u(t)\right) p(t, a) & 0<a<2 \tau \\
p(t, 0)=(1-k(t)) \beta R(t) &
\end{array}
$$

The equation (2.2) is a balance equation for the resting phase between the outward and inward flow; the transport equation (2.3) describes the evolution of the age cohorts of proliferating cells, since they are aging with velocity 1 ; the boundary condition (2.4) gives the inward flow to the proliferating phase.

\subsection{A Controlled Version of Mackey's Model}

We denote by $P$ and $P_{2}$ the total proliferating population and sub-population in the second-half of the phase, respectively:

$$
P(t):=\int_{0}^{2 \tau} p(t, a) \mathrm{d} a, \quad P_{2}(t):=\int_{\tau}^{2 \tau} p(t, a) \mathrm{d} a .
$$

Formally, and this could be justified with the results of Section 3, if we differentiate $P, P_{2}$ and use the method of characteristics as in [1], we derive from (2.1)-(2.4) the following system of delay differential equations:

$$
\begin{aligned}
& \frac{\mathrm{d} R}{\mathrm{~d} t}(t)=-(1-k(t)) \beta R(t)+2(1-k(t-2 \tau)) \beta R(t-2 \tau) e^{-\left(\gamma 2 \tau+\int_{t-\tau}^{t} u(s) \mathrm{d} s\right)} \\
& \frac{\mathrm{d} P}{\mathrm{~d} t}(t)=-\left(\gamma P(t)+u(t) P_{2}(t)\right)+(1-k(t)) \beta R(t) \\
&-(1-k(t-2 \tau)) \beta R(t-2 \tau) e^{-\left(\gamma 2 \tau+\int_{t-\tau}^{t} u(s) \mathrm{d} s\right)} \\
& \frac{\mathrm{d} P_{2}}{\mathrm{~d} t}(t)=-(\gamma+u(t)) P_{2}(t)+(1-k(t-\tau)) \beta R(t-\tau) e^{-\gamma \tau} \\
&-(1-k(t-2 \tau)) \beta R(t-2 \tau) e^{-\left(\gamma 2 \tau+\int_{t-\tau}^{t} u(s) \mathrm{d} s\right)} \\
& \frac{\mathrm{d} k}{\mathrm{~d} t}(t)=v(t)(1-k(t))-\alpha k(t)
\end{aligned}
$$

Unsurprisingly, we get a controlled version of Mackey's 1978 model [18]. The original model is a system of two differential equations with one discrete delay, and a nonlinearity in $\beta$; it is one of the first mathematical model of the dynamics of hematopoietic stem cells (HSC), which are at the root of the hematopoiesis, the process of blood production. We have in (2.5)-(2.8) two control variables, $u$ and $v$, and two extra state variables, $P_{2}$ and $k$, because of the controls.

As we will explain in Section 4.1, the age-structure in the proliferating population actually matters, and thus it is of interest to analyse the age-structured model (2.2)-(2.4). 


\section{Analysis of the Age-Structured Model}

\subsection{Existence of Solutions}

Given $(\beta, \gamma) \in L_{l o c}^{\infty}(0, \infty) \times L_{l o c}^{\infty}((0, \infty) \times(0,2 \tau))$ and $\left(R_{0}, p_{0}\right) \in \mathbb{R} \times L^{\infty}(0,2 \tau)$, we consider the system

$$
\begin{array}{ll}
\frac{\mathrm{d} R}{\mathrm{~d} t}(t)=-\beta(t) R(t)+2 p(t, 2 \tau) & 0<t \\
\frac{\partial p}{\partial t}(t, a)+\frac{\partial p}{\partial a}(t, a)=-\gamma(t, a) p(t, a) & 0<t, 0<a<2 \tau \\
p(t, 0)=\beta(t) R(t) & 0<t
\end{array}
$$

with the initial condition

$$
R(0)=R_{0}, \quad p(0, \cdot)=p_{0} .
$$

We follow [10] for the notion of solution.

Definition 3.1. We say that (3.2) holds along the characteristics a.e. if and only if there holds, for a.a. $(t, a) \in(0, \infty) \times(0,2 \tau)$,

$$
\begin{array}{ll}
p(s, a+s)=p(0, a)-\int_{0}^{s}(\gamma p)(\theta, a+\theta) \mathrm{d} \theta & \text { for a.a. } s \in(0,2 \tau-a), \\
p(t+s, s)=p(t, 0)-\int_{0}^{s}(\gamma p)(t+\theta, \theta) \mathrm{d} \theta & \text { for a.a. } s \in(0,2 \tau) .
\end{array}
$$

Lemma 3.2. If $p \in L_{l o c}^{\infty}((0, \infty) \times(0,2 \tau))$ is such that (3.2) holds along the characteristics a.e., then $p$ is Lipschitz along the characteristics $\{t-a=c\}$ for a.a. $c, t \mapsto \int_{0}^{2 \tau} p(t, a) \mathrm{d} a$ is locally Lipschitz and there holds a.e.

$$
\frac{\mathrm{d}}{\mathrm{d} t} \int_{0}^{2 \tau} p(t, a) \mathrm{d} a=p(t, 0)-p(t, 2 \tau)-\int_{0}^{2 \tau}(\gamma p)(t, a) \mathrm{d} a .
$$

Proof. The first assertion follows from (3.5)-(3.6). For the last two assertions, it is enough to compute $\int_{0}^{2 \tau} p(t, a) \mathrm{d} a$ using the same relations.

Definition 3.3. A solution of (3.1)-(3.4) is any

$$
(R, p) \in W_{l o c}^{1, \infty}(0, \infty) \times L_{l o c}^{\infty}((0, \infty) \times(0,2 \tau))
$$

such that (3.1) holds a.e., (3.2) holds along the characteristics a.e., (3.3) holds a.e., and (3.4) holds.

Lemma 3.4. Given any $(\beta, \gamma)$ and $\left(R_{0}, p_{0}\right)$, there exists a unique solution $(R, p)$ of $(3.1)-(3.4)$. If $(\beta, \gamma)$ and $\left(R_{0}, p_{0}\right)$ are non-negative, then $(R, p)$ is non-negative. Moreover, defining

$$
\Gamma:(t, a) \mapsto \begin{cases}\int_{0}^{t} \gamma(s, a-t+s) \mathrm{d} s & \text { if } 0<t<a<2 \tau, \\ \int_{0}^{a} \gamma(t-a+s, s) \mathrm{d} s & \text { if } 0<a<2 \tau, a<t,\end{cases}
$$

if $\beta, \Gamma, p_{0}$ are locally Lipschitz and $p_{0}(0)=\beta(0) R_{0}$, then $p$ is locally Lipschitz and $R \in W_{\text {loc }}^{2, \infty}$. 
Proof. For a.a. $c \in(-2 \tau, 0), p$ is determined on $\{t-a=c\}$ by

$$
p(t, a)=p_{0}(a-t) e^{-\int_{0}^{t} \gamma(s, a-t+s) \mathrm{d} s} .
$$

Then (3.1) becomes a linear ODE on $(0,2 \tau)$, from which we get $R$, and then $p$ on $\{t-a=c\}$ for a.a. $c \in(0,2 \tau)$, and so on. The sign of $(R, p)$ follows.

Observe that a.e. on $\{t-a>0\}$,

$$
p(t, a)=\beta(t-a) R(t-a) e^{-\int_{0}^{a} \gamma(t-a+s, s) \mathrm{d} s} .
$$

The continuity of $p$ on $\{t-a=0\}$ is equivalent to $p_{0}(0)=\beta(0) R_{0}$.

\subsection{General Relative Entropy}

We introduce the dual system associated with (3.1)-(3.3)

$$
\begin{array}{ll}
\frac{\mathrm{d} \Psi}{\mathrm{d} t}(t)=\beta(t) \Psi(t)-\beta(t) \phi(t, 0) & 0<t \\
\frac{\partial \phi}{\partial t}(t, a)+\frac{\partial \phi}{\partial a}(t, a)=\gamma(t, a) \phi(t, a) & 0<t, 0<a<2 \tau \\
\phi(t, 2 \tau)=2 \Psi(t) & 0<t
\end{array}
$$

Solutions of (3.7)-(3.9) are defined as for the primal system, see Definition 3.3.

Adapting [20] to our model, we get a general relative entropy principle. Let $(\beta, \gamma)$ be fixed. Given a solution $(R, p)$ and a positive solution $(\hat{R}, \hat{p})$ of $(3.1)-(3.3)$, a positive solution $(\Psi, \phi)$ of $(3.7)-(3.9)$, and $H \in L_{\text {loc }}^{\infty}(\mathbb{R})$, we define $\mathcal{H}$ by

$$
\mathcal{H}(t):=\Psi(t) \hat{R}(t) H\left(\frac{R(t)}{\hat{R}(t)}\right)+\int_{0}^{2 \tau} \phi(t, a) \hat{p}(t, a) H\left(\frac{p(t, a)}{\hat{p}(t, a)}\right) \mathrm{d} a .
$$

Theorem 3.5 (General Relative Entropy). Let $H$ be locally Lipschitz and differentiable everywhere. Then $\mathcal{H}$ is locally Lipschitz and there holds a.e.

$$
\frac{\mathrm{d} \mathcal{H}}{\mathrm{d} t}(t)=\phi(t, 2 \tau) \hat{p}(t, 2 \tau)\left[H\left(\frac{p(t, 0)}{\hat{p}(t, 0)}\right)-H\left(\frac{p(t, 2 \tau)}{\hat{p}(t, 2 \tau)}\right)+H^{\prime}\left(\frac{p(t, 0)}{\hat{p}(t, 0)}\right)\left(\frac{p(t, 2 \tau)}{\hat{p}(t, 2 \tau)}-\frac{p(t, 0)}{\hat{p}(t, 0)}\right)\right] .
$$

Corollary 3.6. Let $H$ be convex, possibly non differentiable. Then $\mathcal{H}$ is non-increasing.

Proof of Corollary 3.6. Let $H$ be convex. Then $H$ is locally Lipschitz and has left and right derivatives everywhere. Then, as in the proof of Theorem 3.5, $\mathcal{H}$ is locally Lipschitz and (3.10) holds a.e. if we replace the derivative of $\mathcal{H}$ by its right derivative and the derivative of $H$ by its left or right derivative, depending on the sign of the right derivative of $\frac{p(t, 0)}{\hat{p}(t, 0)}$. Observe now that this right-hand side of (3.10) is non-positive if $H$ is convex.

Proof of Theorem 3.5. Observe that, along the characteristics a.e., there holds

$$
\left(\frac{\partial}{\partial t}+\frac{\partial}{\partial a}\right) \phi(t, a) \hat{p}(t, a)=0, \quad\left(\frac{\partial}{\partial t}+\frac{\partial}{\partial a}\right) \frac{p(t, a)}{\hat{p}(t, a)}=0
$$

and then

$$
\left(\frac{\partial}{\partial t}+\frac{\partial}{\partial a}\right) \phi(t, a) \hat{p}(t, a) H\left(\frac{p(t, a)}{\hat{p}(t, a)}\right)=0 .
$$


By Lemma 3.2, the second term of $\mathcal{H}$ is locally Lipschitz, with derivative a.e.

$$
\phi(t, 0) \hat{p}(t, 0) H\left(\frac{p(t, 0)}{\hat{p}(t, 0)}\right)-\phi(t, 2 \tau) \hat{p}(t, 2 \tau) H\left(\frac{p(t, 2 \tau)}{\hat{p}(t, 2 \tau)}\right) .
$$

The first term of $\mathcal{H}$ is obviously locally Lipschitz, and a.e.

$$
\begin{aligned}
\left(\frac{\mathrm{d}}{\mathrm{d} t} \Psi(t) \hat{R}(t)\right) H\left(\frac{R(t)}{\hat{R}(t)}\right) & =(-\beta(t) \phi(t, 0) \hat{R}(t)+\Psi(t) 2 \hat{p}(t, 2 \tau)) H\left(\frac{R(t)}{\hat{R}(t)}\right) \\
& =(-\phi(t, 0) \hat{p}(t, 0)+\phi(t, 2 \tau) \hat{p}(t, 2 \tau)) H\left(\frac{p(t, 0)}{\hat{p}(t, 0)}\right),
\end{aligned}
$$

and

$$
\begin{aligned}
\Psi(t) \hat{R}(t) \frac{\mathrm{d}}{\mathrm{d} t} H\left(\frac{R(t)}{\hat{R}(t)}\right) & =\Psi(t) \hat{R}(t) H^{\prime}\left(\frac{R(t)}{\hat{R}(t)}\right) \frac{1}{\hat{R}(t)}\left(\frac{\mathrm{d} R}{\mathrm{~d} t}(t)-\frac{R(t)}{\hat{R}(t)} \frac{\mathrm{d} \hat{R}}{\mathrm{~d} t}(t)\right) \\
& =\Psi(t) H^{\prime}\left(\frac{p(t, 0)}{\hat{p}(t, 0)}\right)\left(2 p(t, 2 \tau)-\frac{p(t, 0)}{\hat{p}(t, 0)} 2 \hat{p}(t, 2 \tau)\right) \\
& =\phi(t, 2 \tau) \hat{p}(t, 2 \tau) H^{\prime}\left(\frac{p(t, 0)}{\hat{p}(t, 0)}\right)\left(\frac{p(t, 2 \tau)}{\hat{p}(t, 2 \tau)}-\frac{p(t, 0)}{\hat{p}(t, 0)}\right) .
\end{aligned}
$$

\subsection{Eigenelements}

Let $\beta>0$ and $\gamma \geq 0$ be constant. Looking for particular solutions of (3.1)-(3.3) and (3.7)-(3.9) of the form

$$
\begin{array}{ll}
R: t \mapsto \bar{R} e^{\lambda t} & \Psi: t \mapsto \bar{\Psi} e^{-\lambda t} \\
p:(t, a) \mapsto \bar{p}(a) e^{\lambda t} & \phi:(t, a) \mapsto \bar{\phi}(a) e^{-\lambda t}
\end{array}
$$

with $\lambda \in \mathbb{R}$ and $\bar{p}, \bar{\phi}$ differentiable, we get the following eigenvalue problem:

$$
\begin{array}{ll}
(\lambda+\beta) \bar{R}=2 \bar{p}(2 \tau) & (\lambda+\beta) \bar{\Psi}=\beta \bar{\phi}(0) \\
\frac{\mathrm{d} \bar{p}}{\mathrm{~d} a}(a)=-(\lambda+\gamma) \bar{p}(a) & \frac{\mathrm{d} \bar{\phi}}{\mathrm{d} a}(a)=(\lambda+\gamma) \bar{\phi}(a) \\
\bar{p}(0)=\beta \bar{R} & \bar{\phi}(2 \tau)=2 \bar{\Psi}
\end{array}
$$

Equations (3.12)-(3.13) give

$$
\bar{p}(a)=\beta \bar{R} e^{-(\lambda+\gamma) a}, \quad \bar{\phi}(a)=2 \bar{\Psi} e^{(\lambda+\gamma)(a-2 \tau)} .
$$

If $\bar{R}, \bar{\Psi} \neq 0,(3.11)$ is then equivalent to

$$
\lambda+\beta=2 \beta e^{-(\lambda+\gamma) 2 \tau}
$$

Theorem 3.7 (First eigenelements). There exists a unique solution $(\lambda, \bar{R}, \bar{p}, \bar{\Psi}, \bar{\phi})$ of (3.11)-(3.13) such that

$$
\begin{gathered}
\bar{R}>0, \quad \bar{p}>0, \quad \bar{R}+\int_{0}^{2 \tau} \bar{p}(a) \mathrm{d} a=1, \\
\bar{\Psi}>0, \quad \bar{\phi}>0, \quad \bar{\Psi} \bar{R}+\int_{0}^{2 \tau} \bar{\phi}(a) \bar{p}(a) \mathrm{d} a=1 .
\end{gathered}
$$

Proof. It is enough to observe that (3.15) has a unique real solution. 


\subsection{Long Time Asymptotics}

\subsubsection{Without the Action of the Drugs}

We consider here that there is no action of the drugs for $t>0$, i.e. that $\beta>0$ and $\gamma \geq 0$ are constant. The first eigenelements $(\lambda, \bar{R}, \bar{p}, \bar{\Psi}, \bar{\phi})$ are given by Theorem 3.7 .

Theorem 3.8. Let $\left(R_{0}, p_{0}\right)$ be an initial condition, $C>0$ be such that

$$
\left|R_{0}\right| \leq C \bar{R}, \quad\left|p_{0}(\cdot)\right| \leq C \bar{p}(\cdot),
$$

and $(R, p)$ be the solution of (3.1)-(3.4). Then, for all $t>0$,

$$
\begin{gathered}
|R(t)| \leq C \bar{R} e^{\lambda t}, \quad|p(t, \cdot)| \leq C \bar{p}(\cdot) e^{\lambda t} \\
\left(\bar{\Psi} R(t)+\int_{0}^{2 \tau} \bar{\phi}(a) p(t, a) \mathrm{d} a\right) e^{-\lambda t}=\bar{\Psi} R_{0}+\int_{0}^{2 \tau} \bar{\phi}(a) p_{0}(a) \mathrm{d} a:=\rho, \\
\left(\bar{\Psi}|R(t)|+\int_{0}^{2 \tau} \bar{\phi}(a)|p(t, a)| \mathrm{d} a\right) e^{-\lambda t} \leq \bar{\Psi}\left|R_{0}\right|+\int_{0}^{2 \tau} \bar{\phi}(a)\left|p_{0}(a)\right| \mathrm{d} a \\
\lim _{t \rightarrow \infty}\left(\bar{\Psi}\left|R(t) e^{-\lambda t}-\rho \bar{R}\right|+\int_{0}^{2 \tau} \bar{\phi}(a)\left|p(t, a) e^{-\lambda t}-\rho \bar{p}(a)\right| \mathrm{d} a\right)=0 .
\end{gathered}
$$

Remark 3.9. Theorem 3.8 gives an interpretation of the first eigenelements: for the $L^{1}$ topology,

$$
(R(t), p(t, \cdot)) \sim \rho\left(\bar{R} e^{\lambda t}, \bar{p}(\cdot) e^{\lambda t}\right)
$$

as $t \rightarrow \infty$, with $\rho$ given by (3.17). Then the first eigenvalue $\lambda$ is the Malthus parameter of the model, which gives the overall exponential growth or decay of the population. We derive from its definition (3.15) that $\lambda$ has the same sign as $2 e^{-\gamma 2 \tau}-1$, which is the proliferating phase balance. Asymptotically, any solution becomes proportional to a particular solution with age profile given by the first primal eigenvector $(\bar{R}, \bar{p})$ and rate of time evolution $\lambda$. The coefficient of proportionality $\rho$ is determined initially with the first dual eigenvector $(\bar{\Psi}, \bar{\phi})$.

Proof. We follow the same scheme as in $[20,23]$. We apply the general relative entropy principle to $(R, p),\left(\bar{R} e^{\lambda t}, \bar{p} e^{\lambda t}\right),\left(\bar{\Psi} e^{-\lambda t}, \bar{\phi} e^{-\lambda t}\right)$, and to the following convex functions:

- $H(h):=(h \pm C)_{\mp}^{2}$ for (3.16). The two corresponding entropies $\mathcal{H}$ are non-increasing by Corollary 3.6, non-negative, and initially null; then they are null everywhere.

$-H(h):=h$ for $(3.17) ; \mathcal{H}$ is constant by Theorem 3.5.

$-H(h):=|h|$ for $(3.18) ; \mathcal{H}$ is non-increasing by Corollary 3.6.

$-H(h):=|h-\rho|$ for $(3.19) ; \mathcal{H}$ is non-increasing by Corollary 3.6 and non-negative. Then it has a limit $L$, i.e.

$$
\left(\bar{\Psi}\left|R(t) e^{-\lambda t}-\rho \bar{R}\right|+\int_{0}^{2 \tau} \bar{\phi}(a)\left|p(t, a) e^{-\lambda t}-\rho \bar{p}(a)\right| \mathrm{d} a\right) \rightarrow L
$$

as $t \rightarrow \infty$. It remains to prove that $L=0$; we do that in several steps.

1. Let $p_{0}^{k}, k \in \mathbb{N}$, be Lipschitz, with $p_{0}^{k}(0)=\beta R_{0}$ and such that, as $k \rightarrow \infty$,

$$
\varepsilon_{k}:=\int_{0}^{2 \tau} \bar{\phi}(a)\left|p_{0}^{k}(a)-p_{0}(a)\right| \mathrm{d} a \rightarrow 0 .
$$


Let $\left(R^{k}, p^{k}\right)$ be the solution of (3.1)-(3.3) with initial condition $\left(R_{0}, p_{0}^{k}\right), \rho_{k}$ be given by (3.17), and $L_{k}$ be given by (3.20). Then $\left|\rho_{k}-\rho\right| \leq \varepsilon_{k}$, and applying (3.18) to the solution $\left(R^{k}-R, p^{k}-p\right)$ of (3.1)-(3.3), we get

$$
\left(\bar{\Psi}\left|R^{k}(t)-R(t)\right|+\int_{0}^{2 \tau} \bar{\phi}(a)\left|p^{k}(t, a)-p(t, a)\right| \mathrm{d} a\right) e^{-\lambda t} \leq \varepsilon_{k}
$$

for all $t$. Then $L \leq L_{k}+2 \varepsilon_{k}$, and it is enough to show that $L=0$ for an initial condition $\left(R_{0}, p_{0}\right)$ with $p_{0}$ Lipschitz and $p_{0}(0)=\beta R_{0}$, as we assume in the sequel of the proof.

2. Since $\beta$ and $\gamma$ are constant, $(R, p) \in W_{l o c}^{2, \infty} \times W_{l o c}^{1, \infty}$ by Lemma 3.4. We observe moreover that $\left(\frac{\mathrm{d} R}{\mathrm{~d} t}, \frac{\partial p}{\partial t}\right)$ is a solution of (3.1)-(3.3). Then by (3.16), there exists $C^{\prime}>0$ such that for all $t$,

$$
\left|\frac{\mathrm{d} R}{\mathrm{~d} t}(t)\right| \leq C^{\prime} \bar{R} e^{\lambda t}, \quad\left|\frac{\partial p}{\partial t}(t, \cdot)\right| \leq C^{\prime} \bar{p}(\cdot) e^{\lambda t} .
$$

It follows by (3.2) that for all $t$,

$$
\left|\frac{\partial p}{\partial a}(t, \cdot)\right| \leq\left(\gamma C+C^{\prime}\right) \bar{p}(\cdot) e^{\lambda t} .
$$

3. We apply again Theorem 3.5 to $(R, p),\left(\bar{R} e^{\lambda t}, \bar{p} e^{\lambda t}\right),\left(\bar{\Psi} e^{-\lambda t}, \bar{\phi} e^{-\lambda t}\right)$, and to $H(h):=(h-1)^{2}$. Using that

$$
H\left(h_{1}\right)-H\left(h_{2}\right)+H^{\prime}\left(h_{1}\right)\left(h_{2}-h_{1}\right)=-\left(h_{1}-h_{2}\right)^{2},
$$

we get for the corresponding $\mathcal{H}$

$$
\frac{\mathrm{d} \mathcal{H}}{\mathrm{d} t}(t)=-\bar{\phi}(2 \tau) \bar{p}(2 \tau)\left(\frac{p(t, 2 \tau) e^{-\lambda t}}{\bar{p}(2 \tau)}-\frac{p(t, 0) e^{-\lambda t}}{\bar{p}(0)}\right)^{2} .
$$

Then $\frac{\mathrm{d} \mathcal{H}}{\mathrm{d} t}$ is globally Lipschitz by (3.16) and (3.21), and non-positive. Since $\mathcal{H}$ is bounded below by 0 , it has a limit and then $\frac{\mathrm{d} \mathcal{H}}{\mathrm{d} t}(t) \rightarrow 0$, i.e.

$$
\frac{p(t, 2 \tau) e^{-\lambda t}}{\bar{p}(2 \tau)}-\frac{p(t, 0) e^{-\lambda t}}{\bar{p}(0)} \rightarrow 0
$$

as $t \rightarrow \infty$.

4. We define $\left(Q^{k}, n^{k}\right) \in C([0,1]) \times C([0,1] \times[0,2 \tau]), k \in \mathbb{N}$, by

$$
Q^{k}(t):=R(t+k) e^{-\lambda(t+k)}, \quad n^{k}(t, a):=p(t+k, a) e^{-\lambda(t+k)} .
$$

We derive from (3.16),(3.21)-(3.22) and Arzelà-Ascoli theorem that there exists $(\bar{Q}, \bar{n})$ such that, up to a subsequence, $\left(Q^{k}, n^{k}\right) \rightarrow(\bar{Q}, \bar{n})$ uniformly. Then for all $t \in[0,1]$,

$$
\begin{gathered}
\bar{\Psi} \bar{Q}(t)+\int_{0}^{2 \tau} \bar{\phi}(a) \bar{n}(t, a) \mathrm{d} a=\rho, \\
\bar{\Psi}|\bar{Q}(t)-\rho \bar{R}|+\int_{0}^{2 \tau} \bar{\phi}(a)|\bar{n}(t, a)-\rho \bar{p}(a)| \mathrm{d} a=L, \\
\frac{\bar{n}(t, 2 \tau)}{\bar{p}(2 \tau)}-\frac{\bar{n}(t, 0)}{\bar{p}(0)}=0
\end{gathered}
$$

by $(3.17),(3.20)$, and $(3.23)$, respectively. Moreover $(\bar{Q}, \bar{n})$ is solution, in the sense of Definition 3.3, of

$$
\begin{array}{ll}
\frac{\mathrm{d} Q}{\mathrm{~d} t}(t)=-(\lambda+\beta) Q(t)+2 n(t, 2 \tau) & 0<t<1 \\
\frac{\partial n}{\partial t}(t, a)+\frac{\partial n}{\partial a}(t, a)=-(\lambda+\gamma) n(t, a) & 0<t<1,0<a<2 \tau \\
n(t, 0)=\beta Q(t) & 0<t<1
\end{array}
$$


Injecting (3.26) and (3.29) into (3.27), we get

$$
\frac{\mathrm{d} \bar{Q}}{\mathrm{~d} t}(t)=[-(\lambda+\beta) \bar{p}(0)+2 \beta \bar{p}(2 \tau)] \frac{\bar{Q}(t)}{\bar{p}(0)}=0
$$

by definition (3.14)-(3.15) of the eigenelements. Then $\bar{Q}$ is constant and

$$
\begin{aligned}
\bar{n}(0, a) & =\lim _{k} p(k, a) e^{-\lambda k} \\
& =\lim _{k} \beta R(k-a) e^{-\lambda(k-a)} e^{-(\lambda+\gamma) a} \\
& =\beta \bar{Q} e^{-(\lambda+\gamma) a} .
\end{aligned}
$$

Solving (3.28) along the characteristics, it comes that

$$
\bar{n}(t, a)=\beta \bar{Q} e^{-(\lambda+\gamma) a}
$$

for all $(t, a) \in[0,1] \times[0,2 \tau]$; in particular, $\bar{n}$ does not depend on $t$. Observe that $(\bar{Q}, \bar{n})$ is proportional to $(\bar{R}, \bar{p})$; by $(3.24),(\bar{Q}, \bar{n})=\rho(\bar{R}, \bar{p})$, and then by $(3.25), L=0$ as was to prove.

\subsubsection{With the Action of the Drugs}

We consider now that the drugs are not administrated for $t>0$ but that they have a residual action. Namely, if at $t=0$ there is a fraction $k_{0} \geq 0$ of inhibited cells among the resting cells, then by (2.1), for $t>0$,

$$
\beta(t)=\left(1-k_{0} e^{-\alpha t}\right) \beta
$$

with $\alpha>0$, and $\gamma \geq 0$ is constant. We continue to use the first eigenelements given by Theorem 3.7, i.e. for $\beta$ constant too.

Lemma 3.10. Assume that the first eigenvalue $\lambda$ is non-negative. Let $\left(R_{0}, p_{0}\right)$ be a non-negative initial condition, $(R, p)$ be the solution of (3.1)-(3.4), and I be defined by

$$
I(t):=\left(\bar{\Psi} R(t)+\int_{0}^{2 \tau} \bar{\phi}(a) p(t, a) \mathrm{d} a\right) e^{-\lambda t} .
$$

Then I is locally Lipschitz, non-increasing, and for all $t>0$,

$$
e^{-\lambda \frac{k_{0}}{\alpha}\left(1-e^{-\alpha t}\right)} I(0) \leq I(t) \leq I(0) .
$$

In particular, $I(t)$ has a limit, say $I_{\infty} \in\left[e^{-\lambda \frac{k_{0}}{\alpha}} I(0), I(0)\right]$, as $t \rightarrow \infty$.

Remark 3.11. 1. If $\lambda \leq 0$, then we can show the reverse inequality:

$$
I(0) \leq I(t) \leq e^{-\lambda \frac{k_{0}}{\alpha}\left(1-e^{-\alpha t}\right)} I(0) .
$$

2. If $k_{0}=0$, then $\beta$ is constant and we recover result (3.17) of Theorem 3.8: $I$ is constantly equal to $\rho$.

3. The first eigenvalue $\lambda$ is still the Malthus parameter of the model, in the sense that

$$
\left(R(t) e^{-\lambda^{\prime} t}, p(t, \cdot) e^{-\lambda^{\prime} t}\right) \rightarrow \begin{cases}0 & \text { if } \lambda^{\prime}>\lambda \\ \infty & \text { if } \lambda^{\prime}<\lambda\end{cases}
$$

as $t \rightarrow \infty$. 
4. If there exists $\rho^{\prime}$ such that, in the sense of (3.19),

$$
(R(t), p(t, \cdot)) \sim \rho^{\prime}\left(\bar{R} e^{\lambda t}, \bar{p}(\cdot) e^{\lambda t}\right)
$$

as $t \rightarrow \infty$, then $\rho^{\prime}=I_{\infty}$.

Proof. We still have, along the characteristics a.e.,

$$
\left(\frac{\partial}{\partial t}+\frac{\partial}{\partial a}\right) \bar{\phi}(a) e^{-\lambda t} p(t, a)=0
$$

Then as in the proof of Theorem 3.5, I is locally Lipschitz. And there holds a.e.,

$$
\begin{aligned}
\frac{\mathrm{d} I}{\mathrm{~d} t}(t)= & \bar{\Psi}(-(\lambda+\beta(t)) R(t)+2 p(t, 2 \tau)) e^{-\lambda t} \\
& +(\bar{\phi}(0) p(t, 0)-\bar{\phi}(2 \tau) p(t, 2 \tau)) e^{-\lambda t} \\
= & -\lambda k_{0} e^{-\alpha t} \bar{\Psi} R(t) e^{-\lambda t}
\end{aligned}
$$

Since $\lambda, k_{0}, R, p \geq 0$ (see Lemma 3.4), we get a.e.

$$
-\lambda k_{0} e^{-\alpha t} I(t) \leq \frac{\mathrm{d} I}{\mathrm{~d} t}(t) \leq 0 .
$$

The result follows.

\section{The Optimal Control Problem}

We fix a time horizon $T>0$ and we consider leukemic cell cultures with varying concentrations of both drugs on $[0, T]$. As explained in Section 2.2, we consider that, in our in vitro model (2.1)-(2.4), we control directly the death rate $u$ due to the cytotoxic and the inhibition rate $v$ due to the cytostatic. Thus we call protocol of drugs administration any $(u, v) \in L^{\infty}\left(0, T ; \mathbb{R}^{2}\right)$ satisfying the following biological bounds:

$$
\left\{\begin{array}{l}
0 \leq u(t) \leq \bar{u} \\
0 \leq v(t) \leq \bar{v}
\end{array} \quad \text { for a.a. } t \in(0, T) .\right.
$$

Note that by Lemma 3.4, given any protocol $(u, v)$, there exists a unique associated state, i.e. $(R, p, k)$ such that (2.1)-(2.4) hold.

We are looking for protocols of drugs administration which are as much efficient as possible, and not too toxic. The notion of efficiency will be handled by the objective function (Section 4.1), and the one of toxicity by the constraints (Section 4.2), in our optimal control problem (Section 4.3).

\subsection{Horizon Effect and Age-Weighted Population}

Since we consider only leukemic cells, an efficient protocol has to aim at the extinction of the total population. Nevertheless, if we try to minimize the total population, i.e. if we consider the problem

$$
\min _{(u, v, R, p, k)}\left(R(T)+\int_{0}^{2 \tau} p(T, a) \mathrm{d} a\right) \quad \text { subject to } \quad(2.1)-(2.4),(4.1),
$$

then we observe a horizon effect: it is always optimal to give no cytostatic $v$ at the end of the experiment, whatever the parameters are. It can be seen numerically and proved theoretically, and it is easily understandable: the resting cells which are introduced into the proliferating phase at time $t \in(T-2 \tau, T)$ will not divide before $T$, but might die, which is not the case if they stay in the resting phase; it is 
therefore optimal to have a high global introduction rate, i.e. a low fraction of inhibited cells $k$, at the end. We end up at time $T$ with a filled proliferating phase, which flows into the resting phase after $T$. If for example the death rate in the proliferating phase is so low that its balance is positive, i.e. that it globally produces cells after division, then the total population for this optimal protocol becomes much larger than for other protocols (see Figure 2), which is not satisfying.

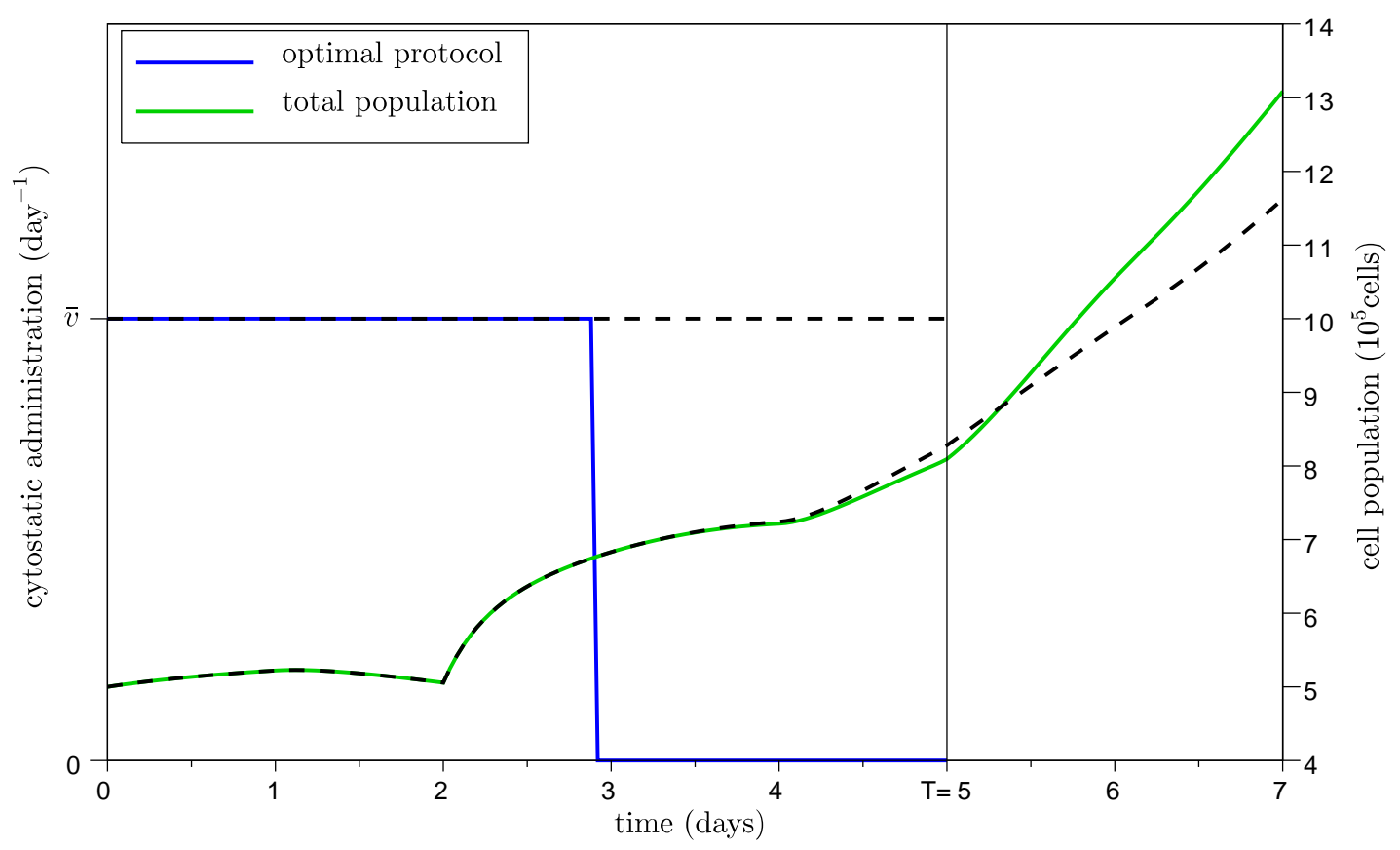

Figure 2. An horizon effect. We consider problem (4.2) with no cytotoxic $u, T=5$ days, $\tau=1$ day; the other parameters are given in Appendix A.3 and are such that the proliferating phase globally produces cells. See Section 5.2 about numerical resolution. The solid lines (resp. the dash lines) represent the optimal protocol (resp. the $\bar{v}$-constant protocol) of cytostatic administration and the associated total population.

Resting cells and proliferating cells with different ages do not have the same role in the population dynamics. Thus it is natural not to give them the same weight in the objective function. One choice of age-dependent weight consists in the first dual eigenvector $(\bar{\Psi}, \bar{\phi})$, given by Theorem 3.7; it is justified by Remarks 3.9 and 3.11. After we stop administrating the drugs at time $T$, there is no action of the cytotoxic and a residual action of the cytostatic, as in Section 3.4.2; nothing can be done on the Malthus parameter $\lambda$, which is given by the uncontrolled system, but we can try to minimize the weighted total population

$$
\bar{\Psi} R(\cdot)+\int_{0}^{2 \tau} \bar{\phi}(a) p(\cdot, a) \mathrm{d} a
$$

at time $T$. If there was no more action of the cytostatic after $T$, the weighted total population (4.3) would be constant for $t>T$ and would give the asymptotic size of the population (Theorem 3.8). It is 
not exactly the case with the residual action of the cytostatic (Lemma 3.10), but even though we choose this weighted total population at time $T$ as the objective function.

\subsection{Maximal Cumulative Doses}

In order to limit the toxicity of the protocols, it is useful to add constraints on the cumulative doses of the drugs. Namely, we fix $\bar{U}, \bar{V}$ and we restrict the optimization problem to the protocols $(u, v)$ such that

$$
\int_{0}^{T} u(t) \mathrm{d} t \leq \bar{U}, \quad \int_{0}^{T} v(t) \mathrm{d} t \leq \bar{V} .
$$

Note that with the bounds (4.1) on the controls, the constraints (4.4) are nontrivial iff

$$
0<\bar{U}<\bar{u} T, \quad 0<\bar{V}<\bar{v} T,
$$

respectively.

\subsection{Reduction to a Problem With Delays}

The state of the cell culture at the beginning of the experiments is fixed; it furnishes the initial condition $\left(k_{0}, R_{0}, p_{0}\right) \in \mathbb{R} \times \mathbb{R} \times L^{\infty}(0,2 \tau)$ of $(2.1)-(2.4)$ :

$$
k(0)=k_{0}, \quad R(0)=R_{0}, \quad p(0, \cdot)=p_{0}
$$

with $k_{0}=0, R_{0}, p_{0} \geq 0$. The issue of finding good protocols of drugs administration can finally be formulated as the following optimal control problem:

$$
\begin{gathered}
\min _{(u, v, R, p, k)}\left(R(T)+\int_{0}^{2 \tau} \bar{\Psi}^{-1} \bar{\phi}(a) p(T, a) \mathrm{d} a\right) \\
\text { subject to }(2.1)-(2.4),(4.1),(4.4)-(4.5) .
\end{gathered}
$$

Recall that $(\bar{\Psi}, \bar{\phi})$ is the first dual eigenvector, defined by Theorem 3.7.

Similarly to the derivation of Mackey's model (Section 2.4), (4.6) can be reduced to an optimal control problem of delay differential equations. We denote by $\tilde{p}, \tilde{P}$ and $\tilde{P}_{2}$ the weighted proliferating population density, the total weighted proliferating population and sub-population in the second-half of the phase, respectively:

$$
\begin{gathered}
\tilde{p}(t, a):=\bar{\Psi}^{-1} \bar{\phi}(a) p(t, a), \\
\tilde{P}(t):=\int_{0}^{2 \tau} \tilde{p}(t, a) \mathrm{d} a, \quad \tilde{P}_{2}(t):=\int_{\tau}^{2 \tau} \tilde{p}(t, a) \mathrm{d} a .
\end{gathered}
$$

Let $(u, v, R, p, k)$ be such that (2.1)-(2.4),(4.5) hold. Observe that, along the characteristics a.e., there holds

$$
\frac{\partial \tilde{p}}{\partial t}(t, a)+\frac{\partial \tilde{p}}{\partial a}(t, a)=\left(\lambda-\chi_{(\tau, 2 \tau)}(a) u(t)\right) \tilde{p}(t, a) .
$$

Then by Lemma 3.2, there holds a.e.

$$
\begin{aligned}
\frac{\mathrm{d} R}{\mathrm{~d} t}(t) & =-(1-k(t)) \beta R(t)+\tilde{p}(t, 2 \tau) \\
\frac{\mathrm{d} \tilde{P}}{\mathrm{~d} t}(t) & =\lambda \tilde{P}(t)-u(t) \tilde{P}_{2}(t)+\tilde{p}(t, 0)-\tilde{p}(t, 2 \tau) \\
\frac{\mathrm{d} \tilde{P}_{2}}{\mathrm{~d} t}(t) & =(\lambda-u(t)) \tilde{P}_{2}(t)+\tilde{p}(t, \tau)-\tilde{p}(t, 2 \tau) \\
\frac{\mathrm{d} k}{\mathrm{~d} t}(t) & =v(t)(1-k(t))-\alpha k(t)
\end{aligned}
$$


where, by definition of $\lambda$ and by the method of characteristics,

$$
\begin{aligned}
\tilde{p}(t, 0) & =(1-k(t))(\lambda+\beta) R(t) \\
\tilde{p}(t, \tau) & = \begin{cases}p_{0}(\tau-t) 2 e^{-\gamma t-(\lambda+\gamma) \tau} & \text { if } t<\tau \\
(1-k(t-\tau))(\lambda+\beta) R(t-\tau) e^{\lambda \tau} & \text { if } t>\tau\end{cases} \\
\tilde{p}(t, 2 \tau) & = \begin{cases}p_{0}(2 \tau-t) 2 e^{-\gamma t-y(t)} & \text { if } t<2 \tau \\
(1-k(t-2 \tau))(\lambda+\beta) R(t-2 \tau) e^{\lambda 2 \tau-y(t)} & \text { if } t>2 \tau\end{cases} \\
\text { with } y(t) & := \begin{cases}\int_{0}^{t} u(s) \mathrm{d} s & \text { if } t<\tau \\
\int_{t-\tau}^{t} u(s) \mathrm{d} s & \text { if } t>\tau\end{cases}
\end{aligned}
$$

We consider $y$ as a new state variable, and we also introduce two extra state variables $U$ and $V$ in order to handle the integral constraints (4.4):

$$
\frac{\mathrm{d} y}{\mathrm{~d} t}(t)=\left\{\begin{array}{ll}
u(t) & \text { if } t<\tau \\
u(t)-u(t-\tau) & \text { if } t>\tau
\end{array}, \quad \frac{\mathrm{d} U}{\mathrm{~d} t}(t)=u(t), \quad \frac{\mathrm{d} V}{\mathrm{~d} t}(t)=v(t) .\right.
$$

Observe that (4.7)-(4.14) is a system of ordinary differential equations for $t<\tau$; it becomes a system of differential equations with one discrete delay for $\tau<t<2 \tau$, and with two discrete delays for $t>2 \tau$. Its initial condition is the following:

$$
\begin{gathered}
R(0)=R_{0}, \quad k(0)=0, \quad y(0)=0, \quad U(0)=0, \quad V(0)=0 \\
\tilde{P}(0)=\int_{0}^{2 \tau} 2 e^{(\lambda+\gamma)(a-2 \tau)} p_{0}(a) \mathrm{d} a, \quad \tilde{P}_{2}(0)=\int_{\tau}^{2 \tau} 2 e^{(\lambda+\gamma)(a-2 \tau)} p_{0}(a) \mathrm{d} a .
\end{gathered}
$$

Problem (4.6) is therefore equivalent to the following optimal control problem:

$$
\min _{\left(u, v, R, \tilde{P}, \tilde{P}_{2}, k, y, U, V\right)}(R+\tilde{P})(T)
$$

subject to (4.7)-(4.15), $\left\{\begin{array}{l}0 \leq u(t) \leq \bar{u} \\ 0 \leq v(t) \leq \bar{v}\end{array}\right.$ for a.a. $t \in(0, T)$, and $\left\{\begin{array}{l}U(T) \leq \bar{U} \\ V(T) \leq \bar{V}\end{array}\right.$.

\section{Results and Conclusion}

We present in this section some theoretical and numerical results on the optimal control problem introduced in the previous section. We use either its form (4.6) or (4.16); the data are

$$
T>2 \tau>0, R_{0}, p_{0} \geq 0, \alpha, \beta>0, \gamma \geq 0, \bar{u}, \bar{v}, \bar{U}, \bar{V} \geq 0 .
$$

The first eigenvalue $\lambda$ is determined by (3.15).

\subsection{Existence and Optimality Conditions}

We begin with a result of existence of an optimal protocol of drugs administration. It relies on the fact that the dynamics is affine w.r.t. the controls. We do not have uniqueness in general.

Proposition 5.1. There exists at least one optimal protocol of drugs administration $(\hat{u}, \hat{v})$ with associated state $(\hat{R}, \ldots, \hat{V})$. 
Proof. The value of problem (4.16) is non-negative; let $\left(u^{k}, v^{k}, R^{k}, \ldots, V^{k}\right)$ be a minimizing sequence. Observe that $\left(u^{k}, v^{k}\right)$ is bounded in $L^{\infty}$, and $\left(R^{k}, \ldots, V^{k}\right)$ is bounded and equicontinuous on $[0, T]$. Then by Banach-Alaoglu theorem and Arzelà-Ascoli theorem, there exists $(\hat{u}, \ldots, \hat{V})$ such that, up to a subsequence,

$$
\left(u^{k}, v^{k}\right) \rightarrow(\hat{u}, \hat{v}) \quad \text { and } \quad\left(R^{k}, \ldots, V^{k}\right) \rightarrow(\hat{R}, \ldots, \hat{V})
$$

for the weak $*$ topology in $L^{\infty}$ and the uniform topology in $C^{0}$, respectively. Since the dynamics is affine w.r.t. the controls, $(\hat{u}, \ldots, \hat{V})$ satisfies (4.7)-(4.15). The bounds and the final constraints are also satisfied, and the objective function is minimized by construction.

The second result says that it is optimal to administrate as much of cytotoxic as possible. It implies uniqueness of the optimal protocol of cytotoxic administration when it is not constrained by a maximal cumulative dose. The constrained case will be studied numerically later.

Proposition 5.2. Let $(\hat{u}, \hat{v})$ be an optimal protocol of drugs administration. Then

$$
\int_{0}^{T} \hat{u}(t) \mathrm{d} t=\min \{\bar{u} T, \bar{U}\}
$$

In particular, if $\bar{U} \geq \bar{u} T$, then $\hat{u}(t)=\bar{u}$ a.e. on $(0, T)$.

Proof. If $\hat{U}(T)<\min \{\bar{u} T, \bar{U}\}$, then there exists an admissible $u$ such that $u \geq \hat{u}, u \neq \hat{u}$. The result follows from the fact that (2.2)-(2.4) is monotone w.r.t. $u \in L^{\infty}(0, T)$.

Next we state first-order optimality conditions, in the form of Pontryagin's minimum principle and where we highlight that the dynamics is affine w.r.t. the controls.

Proposition 5.3. Let $(\hat{u}, \hat{v})$ be an optimal protocol of drugs administration. Then there exists $(a, b) \in$ $W^{1, \infty}\left(0, T ; \mathbb{R}^{2}\right)$ such that, for a.a. $t \in(0, T)$,

$$
(\hat{u}(t), \hat{v}(t)) \in \operatorname{argmin}\left\{a(t) u+b(t) v: \begin{array}{l}
0 \leq u \leq \bar{u} \\
0 \leq v \leq \bar{v}
\end{array}\right\}
$$

Proof. We apply Pontryagin's minimum principle to the delayed problem (4.16). It can be done either directly [14], or after Guinn's transformation [12,13] into an optimal control problem of ordinary differential equations [8]. The minimized function is linear w.r.t. $(u, y)$ at all time because the dynamics is affine w.r.t. the controls. See Appendices A.1 and A.2 for the precise statement of Pontryagin's minimum principle and the expression of coefficients $a$ and $b$.

Then we expect, in the sense of the following corollary, the optimal protocols to be bang-bang, i.e. on their bounds.

Corollary 5.4. For a.a. $t \in(0, T)$,

$$
\hat{u}(t)=\left\{\begin{array}{ll}
0 & \text { if } a(t)>0 \\
\bar{u} & \text { if } a(t)<0
\end{array} \quad \text { and } \quad \hat{v}(t)=\left\{\begin{array}{ll}
0 & \text { if } b(t)>0 \\
\bar{v} & \text { if } b(t)<0
\end{array}\right. \text {. }\right.
$$

It is sometimes possible to determine the sign of $b$, and then the value of $\hat{v}$.

Proposition 5.5. Let $\bar{V} \geq \bar{v} T$ and let $(\hat{u}, \hat{v})$ be an optimal protocol of drugs administration. Then there exists $\varepsilon>0$ such that

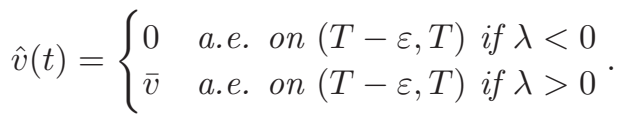

Proof. It is important here to have Pontryagin's minimum principle with normal multipliers. See Appendix A.2 for the determination of the sign of $b$. 


\subsection{Optimal Protocols}

We use BOCOP [9] to solve numerically the undelayed optimal control problem obtained by Guinn's transformation $[12,13]$ of the delayed problem $(4.16)$. We discuss here the optimal protocol $(\hat{u}, \hat{v})$, with associated state $(\hat{R}, \ldots, \hat{V})$, found numerically in different situations; we define the minimal and maximal proliferating phase balances respectively by

$$
\delta_{\bar{u}}:=2 e^{-(\gamma 2 \tau+\bar{u} \tau)}-1, \quad \delta_{0}:=2 e^{-\gamma 2 \tau}-1 .
$$

Note that $\delta_{\bar{u}} \leq \delta_{0}$, the latter having the same sign as $\lambda$ by (3.15).

The case $\delta_{0} \leq 0$ corresponds to a situation where the proliferating phase globally kills cells, even without the administration of any cytotoxic; $\lambda \leq 0$ and then there is no natural growth of the leukemic cell population: this is not a cancer situation. It could be seen that in this case, it is optimal to give no cytostatic: $\hat{v}(t)=0$ a.e. on $(0, T)$, because the higher the global introduction rate, the greater the loss of cells.

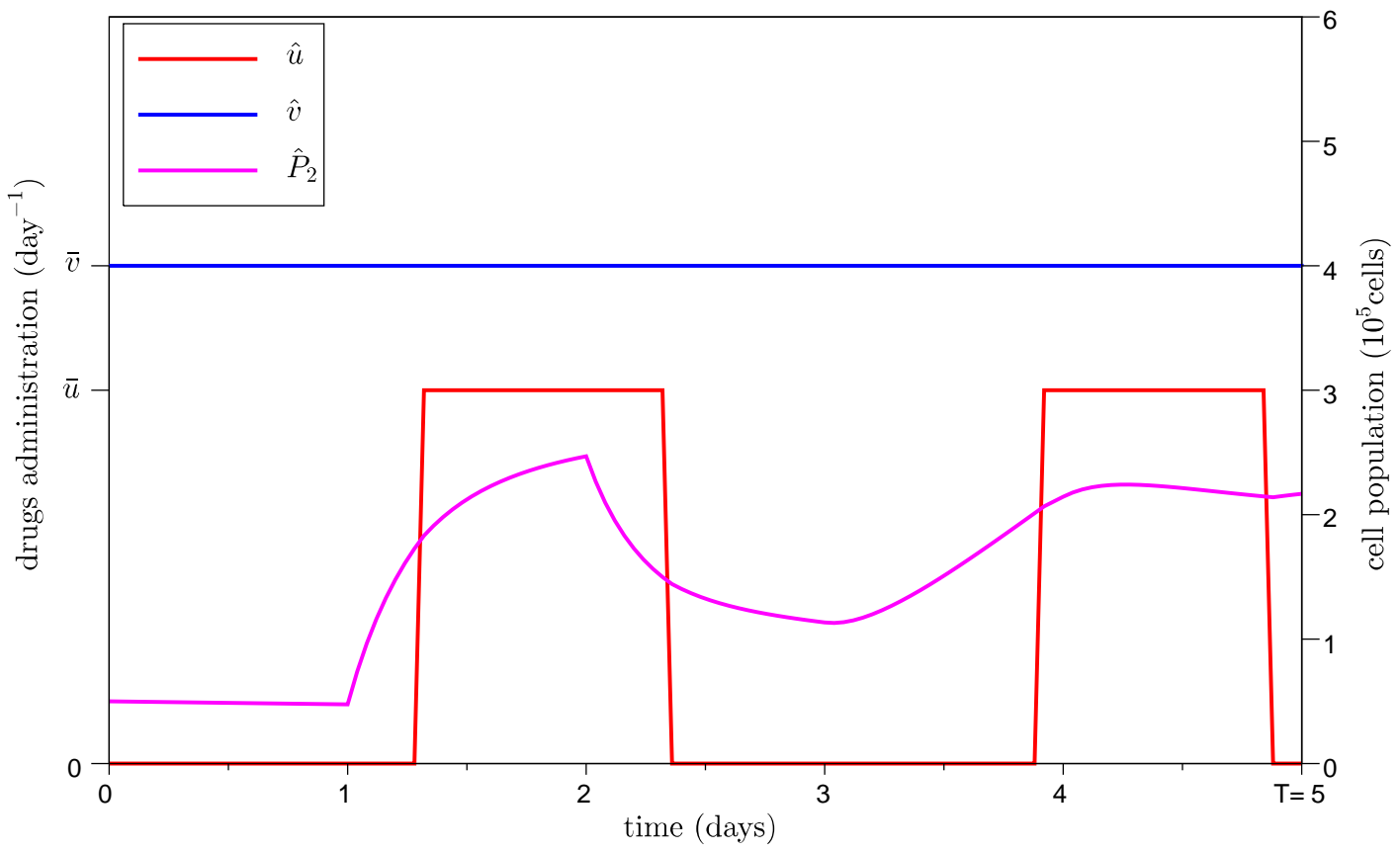

Figure 3. An optimal protocol with $0 \leq \delta_{\bar{u}}$ and limited cytotoxic. We consider a maximal cumulative dose of cytotoxic $\bar{U}=2$ days $\cdot \bar{u}$, whereas $T=5$ days, $\tau=1$ day; the other parameters are given in Appendix A.3. In addition to the optimal protocol of drugs administration $(\hat{u}, \hat{v})$, the associated total sub-population $\hat{P}_{2}$ is plotted.

The case $0 \leq \delta_{\bar{u}}$ corresponds to a situation where the proliferating phase globally produces cells, even with the administration of a maximum of cytotoxic; this is a very severe cancer situation. By Lemma 5.2, it is optimal to administrate as much of cytotoxic as possible; therefore we consider a nontrivial constraint (4.4) on the cumulative dose of cytotoxic with $0<\bar{U}<\bar{u} T$, and no constraint on the cytostatic. We observe in Figure 3 that the optimal protocol of cytotoxic administration is bang-bang, with $\hat{u}(t)=\bar{u}$ 
a.e. when the total sub-population $\hat{P}_{2}$ (on which the cytotoxic is acting) is relatively high. And contrary to Section 4.1 and Figure 2, the optimal protocol of cytostatic administration is now $\hat{v}(t)=\bar{v}$ a.e. on $(0, T)$, because the lower the global introduction rate, the smaller the gain of cells.

The case $\delta_{\bar{u}}<0<\delta_{0}$ corresponds to a situation where the proliferating phase globally produces cells in absence of drugs, and the administration of cytotoxic can make it globally kill cells; this is the most interesting situation.

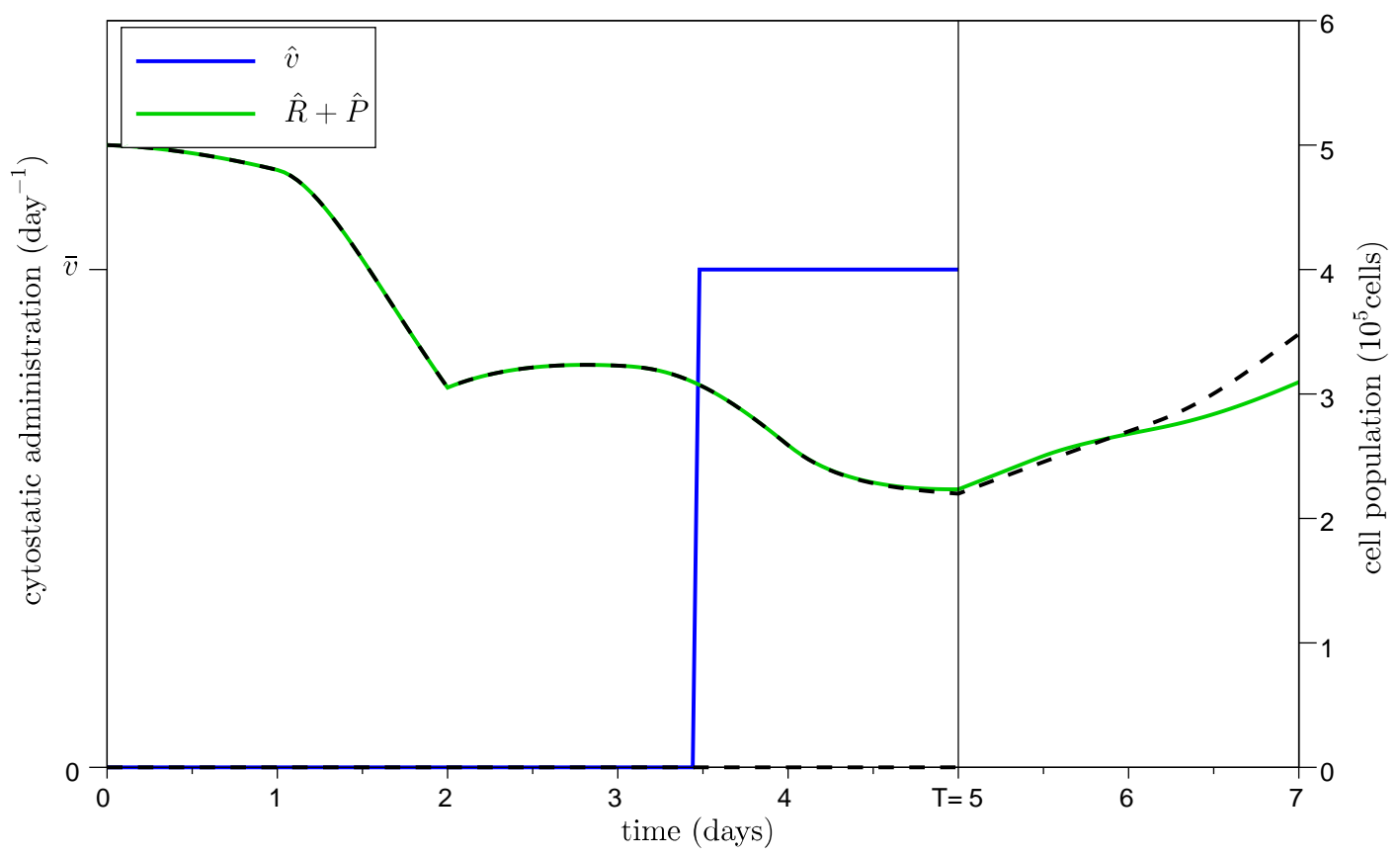

Figure 4. An optimal protocol of with $\delta_{\bar{u}}<0<\delta_{0}$. We consider no constraint on the cumulative dose of cytotoxic, $T=5$ days, $\tau=1$ day; the other parameters are given in Appendix A.3. The protocol of cytotoxic administration is fixed to the optimal $\hat{u}(t)=\bar{u}$ a.e. on $(0, T)$ and is not plotted; the solid lines (resp. the dash lines) represent the optimal protocol (resp. the 0-constant protocol) of cytostatic administration and the associated total population.

First we consider no constraint on the cumulative doses of the drugs. The optimal protocol of cytotoxic administration is again $\hat{u}(t)=\bar{u}$ a.e. on $(0, T)$ and we do not plot it in Figure 4 . We observe that the optimal protocol of cytostatic administration is bang-bang, with $\hat{v}(t)=0$ a.e. first and $\hat{v}(t)=\bar{v}$ a.e. second. For comparison, we also plot the 0 -constant protocol and the associated total population, which is slightly lower than for the optimal protocol at time $T$, but quickly becomes higher. The switch in the optimal protocol of cytostatic administration can be understood as follows: the resting cells which are introduced into the proliferating phase at time $t \in(0, T-2 \tau)$ will have a proliferating phase whose balance is $\delta_{\bar{u}}<0$, whereas those introduced at time $t \in(T-\tau, T)$ will have a proliferating phase whose balance is $\delta_{0}>0$; it is therefore of interest to have a high global introduction rate during $(0, T-2 \tau)$ and a low one during $(T-\tau, T)$. Recall that we do not control directly the fraction of inhibited cells $k$, but the inhibition rate $v$. Note that by Proposition 5.5, we expected to have $\hat{v}(t)=\bar{v}$ a.e. at the end. 


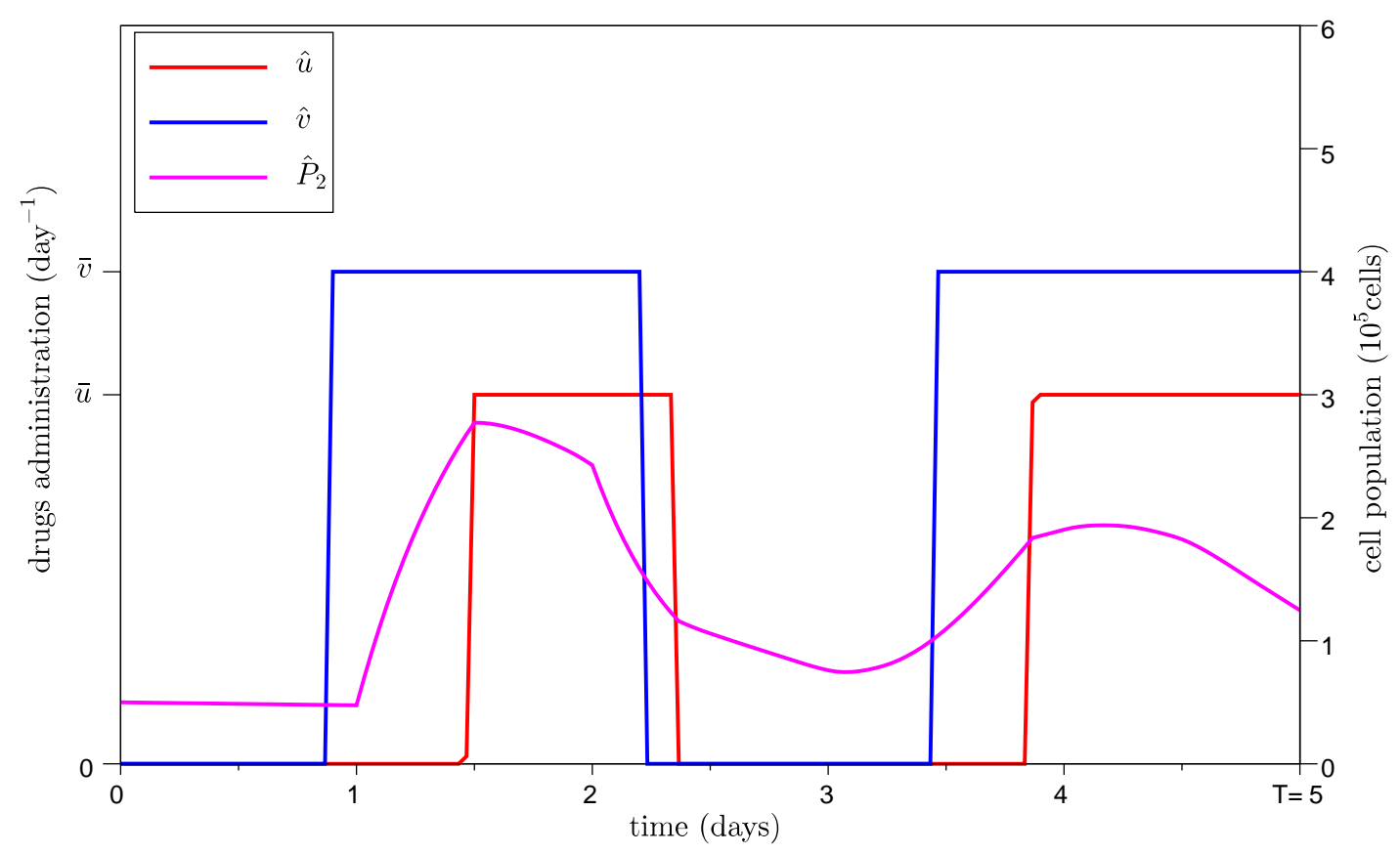

Figure 5. An optimal protocol with $\delta_{\bar{u}}<0<\delta_{0}$ and limited cytotoxic. We consider a maximal cumulative dose of cytotoxic $\bar{U}=2$ days $\cdot \bar{u}$, whereas $T=5$ days, $\tau=1$ day; the other parameters are given in Appendix A.3. In addition to the optimal protocol of drugs administration $(\hat{u}, \hat{v})$, the associated total sub-population $\hat{P}_{2}$ is plotted.

Second we add a nontrivial constraint on the cumulative dose of cytotoxic only, as in the case of Figure 3. Again, the optimal protocol of cytotoxic administration in Figure 5 is bang-bang, with $\hat{u}(t)=\bar{u}$ a.e. when the total sub-population $\hat{P}_{2}$ is relatively high. The optimal protocol of cytostatic administration $\hat{v}$ is also bang-bang, and its structure can be understood similarly to one of Figure 4: it is of interest to have a low global introduction rate if the cells which are just introduced are going to have a proliferating phase whose balance is positive, in particular if a time $\tau$ later, $\hat{u}=0$ a.e. on a long enough interval; and it is also of interest to have a high global introduction rate a time $\tau$ before the intervals where $\hat{u}=\bar{u}$ a.e., in order for the cytotoxic to be efficient. Note that in this interpretation, $\hat{u}$ depends on $\hat{P}_{2}$, which depends on $\hat{v}$, which depends on $\hat{u}$.

\subsection{Conclusion}

The issue of finding good protocols of drugs administration in leukemic cell cultures has been formulated as an optimal control problem, where the population dynamics is eventually reduced to a delay differential system. It has to be noted that the definition of the objective function for this optimization problem is a nontrivial part of the modeling, and that it might still be improved if we could find explicitly the limit $I_{\infty}$ in Lemma 3.10. This approach is different from [7], where the objective function is the Floquet eigenvalue of a periodic problem.

A few optimal protocols have been presented to illustrate different behaviors, which are not always intuitive. Optimal protocols in general have not been synthesized; the dimension 7 of the differential 
system and the fact that the adjoint state equations are with advanced arguments have to be added to the complexity described in [16] for combined treatments. Nevertheless, it is not excluded to get further results on bang-bang and singular controls, as in [16,17], from the analysis started in Appendix A.2.

Estimated parameters are needed for medical application and are to be published [4]. The optimal control problem being set and numerically implemented, it could suggest in vitro protocols to the biologists, and maybe answer questions of the clinicians. It could also simulate experiments longuer than 5 days, which are complicated to carry out for pratical reasons. For in vivo modeling, pharmacokineticspharmacodynamics (PK-PD) would have to be added, as in $[5,16,17]$.

\section{A. Appendix}

\section{A.1. Pontryagin's Minimum Principle}

We consider problem (4.16) and its dynamics (4.7)-(4.14). We denote by $R^{0}, R^{1}$, and $R^{2}$ the arguments of the state variable $R$ with a delay $0, \tau$, and $2 \tau$, respectively; we denote similarly the different arguments of all the undelayed and delayed state and control variables. We then define the following functions of $t$ and

$$
\left(u^{0}, u^{1}, v^{0}, R^{0}, R^{1}, R^{2}, \tilde{P}^{0}, \tilde{P}_{2}^{0}, k^{0}, k^{1}, k^{2}, y^{0}, U^{0}, V^{0}\right)
$$

by

$$
\begin{aligned}
& F_{R}(t, \cdot):= \begin{cases}-\left(1-k^{0}\right) \beta R^{0}+p_{0}(2 \tau-t) 2 e^{-\gamma t-y^{0}} & \text { if } t<2 \tau \\
-\left(1-k^{0}\right) \beta R^{0}+\left(1-k^{2}\right)(\lambda+\beta) R^{2} e^{\lambda 2 \tau-y^{0}} & \text { if } t>2 \tau\end{cases} \\
& F_{\tilde{P}}(t, \cdot):=\left\{\begin{array}{rr}
\lambda \tilde{P}^{0}-u^{0} \tilde{P}_{2}^{0}+\left(1-k^{0}\right)(\lambda+\beta) R^{0} \\
-p_{0}(2 \tau-t) 2 e^{-\gamma t-y^{0}} & \text { if } t<2 \tau \\
\lambda \tilde{P}^{0}-u^{0} \tilde{P}_{2}^{0}+\left(1-k^{0}\right)(\lambda+\beta) R^{0} & \\
-\left(1-k^{2}\right)(\lambda+\beta) R^{2} e^{\lambda 2 \tau-y^{0}} & \text { if } t>2 \tau
\end{array}\right. \\
& F_{\tilde{P}_{2}}(t, \cdot):=\left\{\begin{aligned}
\left(\lambda-u^{0}\right) \tilde{P}_{2}^{0}+p_{0}(\tau-t) 2 e^{-\gamma t-(\lambda+\gamma) \tau} & \\
-p_{0}(2 \tau-t) 2 e^{-\gamma t-y^{0}} & \text { if } t<\tau \\
\left(\lambda-u^{0}\right) \tilde{P}_{2}^{0}+\left(1-k^{1}\right)(\lambda+\beta) R^{1} e^{\lambda \tau} & \\
-p_{0}(2 \tau-t) 2 e^{-\gamma t-y^{0}} & \text { if } \tau<t<2 \tau \\
\left(\lambda-u^{0}\right) \tilde{P}_{2}^{0}+\left(1-k^{1}\right)(\lambda+\beta) R^{1} e^{\lambda \tau} & \\
-\left(1-k^{2}\right)(\lambda+\beta) R^{2} e^{\lambda 2 \tau-y^{0}} & \text { if } t>2 \tau
\end{aligned}\right. \\
& \begin{array}{ll}
F_{k}(t, \cdot):=v^{0}\left(1-k^{1}\right)-\alpha k^{0} & F_{y}(t, \cdot):= \begin{cases}u^{0} & \text { if } t<\tau \\
u^{0}-u^{1} & \text { if } t>\tau\end{cases} \\
F_{U}(t, \cdot):=u^{0} & F_{V}(t, \cdot):=v^{0}
\end{array}
\end{aligned}
$$

The system (4.7)-(4.14) can now be written as

$$
\begin{array}{r}
\frac{\mathrm{d} x}{\mathrm{~d} t}(t)=F_{x}\left(t, u(t), u(t-\tau), v(t), R(t), R(t-\tau), R(t-2 \tau), \tilde{P}(t), \tilde{P}_{2}(t),\right. \\
k(t), k(t-\tau), k(t-2 \tau), y(t), U(t), V(t))
\end{array}
$$

for $x \in\left\{R, \tilde{P}, \tilde{P}_{2}, k, y, U, V\right\}$ and for a.a. $t \in(0, T)$. 
We define the Hamiltonian and the final point Lagrangian respectively as follows: given $q=\left(q_{R}, q_{\tilde{P}}, q_{\tilde{P}_{2}}, q_{k}, q_{y}, q_{U}, q_{V}\right) \in W^{1, \infty}\left(0, T ; \mathbb{R}^{7}\right)$, let

$$
H[q](t, \cdot):=\sum_{x} q_{x}(t) F_{x}(t, \cdot), \quad x \in\left\{R, \tilde{P}, \tilde{P}_{2}, k, y, U, V\right\},
$$

and given $\Psi=\left(\Psi_{U}, \Psi_{V}\right) \in \mathbb{R}^{2}$, let

$$
\Phi[\Psi](\cdot):=R^{0}+\tilde{P}^{0}+\Psi_{U}\left(U^{0}-\bar{U}\right)+\Psi_{V}\left(V^{0}-\bar{V}\right),
$$

where $\cdot$ stands again for $\left(u^{0}, u^{1}, v^{0}, R^{0}, R^{1}, R^{2}, \tilde{P}^{0}, \tilde{P}_{2}^{0}, k^{0}, k^{1}, k^{2}, y^{0}, U^{0}, V^{0}\right)$.

Given a protocol of drugs administration $(\hat{u}, \hat{v})$, and $(\hat{R}, \ldots, \hat{V})$ its associated state, we denote by $\widehat{H}[q](t)$ the evalution of $H[q]$ at

$$
(t, \hat{u}(t), \hat{u}(t-\tau), \hat{v}(t), \hat{R}(t), \hat{R}(t-\tau), \hat{R}(t-2 \tau), \ldots, \hat{V}(t)),
$$

by $\widehat{\Phi}[\Psi](T)$ the evaluation of $\Phi[\Psi]$ at

$$
(\hat{u}(T), \hat{u}(T-\tau), \hat{v}(T), \hat{R}(T), \hat{R}(T-\tau), \hat{R}(T-2 \tau), \ldots, \hat{V}(T)),
$$

and similarly for their partial derivatives. We can now state Pontryagin's minimum principle:

Theorem A.1. Let $(\hat{u}, \hat{v})$ be an optimal protocol of drugs administration with associated state $(\hat{R}, \ldots, \hat{V})$. Then there exist $q \in W^{1, \infty}\left(0, T ; \mathbb{R}^{7}\right)$ and $\Psi \in \mathbb{R}^{2}$ such that, for a.a. $t \in(0, T)$,

$$
\begin{aligned}
-\frac{\mathrm{d} q_{x}}{\mathrm{~d} t}(t)= & D_{x^{0}} \widehat{H}[q](t)+\chi_{(0, T-\tau)}(t) D_{x^{1}} \widehat{H}[q](t+\tau) \quad q_{x}(T)=D_{x^{0}} \widehat{\Phi}[\Psi](T) \\
& +\chi_{(0, T-2 \tau)}(t) D_{x^{2}} \widehat{H}[q](t+2 \tau),
\end{aligned}
$$

for $x \in\left\{R, \tilde{P}, \tilde{P}_{2}, k, y, U, V\right\} ;$ for a.a. $t \in(0, T)$,

$$
\begin{aligned}
\widehat{H}[q](t)+\chi_{(0, T-\tau)}(t) \hat{H}[q](t & +\tau) \leq H[q](t, u, \hat{u}(t-\tau), v, \hat{R}(t), \ldots, \hat{V}(t)) \\
& +\chi_{(0, T-\tau)}(t) H[q](t+\tau, \hat{u}(t+\tau), u, \hat{v}(t+\tau), \hat{R}(t+\tau), \ldots, \hat{V}(t+\tau))
\end{aligned}
$$

for all $(u, v) \in[0, \bar{U}] \times[0, \bar{V}]$; and

$$
\begin{aligned}
& \Psi_{U} \geq 0, \quad \Psi_{U}(\hat{U}(T)-\bar{U})=0, \\
& \Psi_{V} \geq 0, \quad \Psi_{V}(\hat{V}(T)-\bar{V})=0 .
\end{aligned}
$$

Proof. This is Pontryagin's minimum principle $[8,12,14]$. Observe that problem (4.16) satisfies a Mangasarian-Fromovitz condition of qualification if $\bar{U}, \bar{V}>0$; we consider the optimal control problem without the control $u$ (resp. $v$ ) if $\bar{U}=0$ (resp. $\bar{V}=0$ ), and it becomes qualified. Then we get the existence of normal multipliers [8].

\section{A.2. Proof of Propositions 5.3 and 5.5}

By (A.1), $q_{U} \equiv \Psi_{U}$ and $q_{V} \equiv \Psi_{V}$. Since $H[q]$ is affine w.r.t. $\left(u^{0}, u^{1}, v^{0}\right)$, we derive Proposition 5.3 from the Hamiltonian minimum condition (A.2), with

$$
\begin{aligned}
a(t) & =D_{u^{0}} \widehat{H}[q](t)+\chi_{(0, T-\tau)}(t) D_{u^{1}} \widehat{H}[q](t+\tau) \\
& =\Psi_{U}-\left(q_{\tilde{P}}(t)+q_{\tilde{P}_{2}}(t)\right) \hat{\tilde{P}}_{2}(t)+q_{y}(t)+\chi_{(0, T-\tau)}(t) q_{y}(t+\tau), \\
b(t) & =D_{v^{0}} \widehat{H}[q](t) \\
& =\Psi_{V}+q_{k}(t)(1-\hat{k}(t)) .
\end{aligned}
$$


For Proposition 5.5, we need to determine the sign of $b$ in a neighborhood of $T$. Let $\bar{V} \geq \bar{v} T$; considering the equivalent optimization problem without the constraint on $V$, we can assume that $\Psi_{V}=0$. Since $1-\hat{k}>0, b$ has then the same sign as $q_{k}$, whose adjoint equation (A.1) is

$$
\begin{aligned}
\frac{\mathrm{d} q_{k}}{\mathrm{~d} t}(t)= & q_{k}(t)(\hat{v}(t)+\alpha)-\left(q_{R}-q_{\tilde{P}}\right)(t) \beta \hat{R}(t)+q_{\tilde{P}}(t) \lambda \hat{R}(t) \\
& +\chi_{(0, T-\tau)}(t) q_{\tilde{P}_{2}}(t+\tau)(\lambda+\beta) \hat{R}(t) e^{\lambda \tau} \\
& +\chi_{(0, T-2 \tau)}(t)\left(q_{R}-q_{\tilde{P}}-q_{\tilde{P}_{2}}\right)(t+2 \tau)(\lambda+\beta) \hat{R}(t) e^{\lambda 2 \tau-\hat{y}(t+\tau)}
\end{aligned}
$$

for a.a. $t \in(0, T)$, and $q_{k}(T)=0$.

Lemma A.2. Let $c, d \in L^{\infty}(\mathbb{R})$ and $w, z \in W_{l o c}^{1, \infty}$ be such that, for a.a. $t$,

$$
\begin{aligned}
& \dot{w}(t)=c(t) w(t)+d(t) \quad w(T)=\bar{w}, \\
& \dot{z}(t)=d(t) e^{\int_{t}^{T} c(\theta) \mathrm{d} \theta} \quad z(T)=\bar{w} .
\end{aligned}
$$

Then $w$ and $z$ have the same sign.

Proof. Simply observe that $w(t)=\left(\bar{w}-\int_{t}^{T} d(s) e^{\int_{s}^{T} c(\theta) \mathrm{d} \theta} \mathrm{d} s\right) e^{-\int_{t}^{T} c(\theta) \mathrm{d} \theta}=z(t) e^{-\int_{t}^{T} c(\theta) \mathrm{d} \theta}$.

Let $f \in L^{\infty}(0, T)$ be defined by

$$
\begin{aligned}
f(t):=-\left(q_{R}-q_{\tilde{P}}\right)(t) \beta+q_{\tilde{P}}(t) \lambda+\chi_{(0, T-\tau)}(t) q_{\tilde{P}_{2}}(t+\tau)(\lambda+\beta) e^{\lambda \tau} & \\
& +\chi_{(0, T-2 \tau)}(t)\left(q_{R}-q_{\tilde{P}}-q_{\tilde{P}_{2}}\right)(t+2 \tau)(\lambda+\beta) e^{\lambda 2 \tau-\hat{y}(t+\tau)}
\end{aligned}
$$

and $\sigma \in W^{1, \infty}(0, T)$ be such that, for a.a. $t$,

$$
\dot{\sigma}(t)=f(t) \hat{R}(t) e^{\int_{t}^{T}(\hat{v}(\theta)+\alpha) \mathrm{d} \theta} \quad \sigma(T)=0 .
$$

Then $b$ has the same sign as $\sigma$. By the final condition of the adjoint equations (A.1), $f(T)=\lambda$. Since $f$ is left-continuous on $T$, there exists $\varepsilon>0$ such that $f$, and then $\dot{\sigma}$, have the same sign as $\lambda$ on $(T-\varepsilon, T)$. Proposition 5.5 follows.

\section{A.3. Parameters for the numerical resolutions}

These parameters have not been estimated; some of them are fixed in coherence with data from the experiments described in the introduction [4], the others are chosen to explore different situations.

Figure 2 The parameters are the following:

$$
\begin{gathered}
T=5 \text { days } \tau=1 \text { day } \\
R_{0}=4 \times 10^{5} \text { cells } \quad p_{0}(a)=0.5 \times 10^{5}{\text { cells } \times \text { day }^{-1}}^{-1} \quad \beta=2 \text { day }^{-1} \quad \bar{v}=2 \text { day }^{-1} \\
\alpha=1 \text { day }^{-1} \\
\gamma=0.15 \text { day }^{-1}
\end{gathered}
$$

The proliferating phase balance is then $2 e^{-\gamma 2 \tau}-1 \approx 0.48>0$.

Figure 3 The parameters are the following: (A.3)-(A.5) and

$$
\gamma=0.05 \text { day }^{-1} \quad \bar{u}=0.2 \text { day }^{-1} \quad \bar{U}=2 \text { days } \cdot \bar{u}
$$

Note that for these values, $0<\delta_{\bar{u}} \approx 0.48<\delta_{0} \approx 0.81$. Solving numerically (3.15), we get $\lambda \approx 0.24$ day $^{-1}>0$. 
Figure 4 The parameters are the following: (A.3)-(A.5) and

$$
\gamma=0.05 \text { day }^{-1} \quad \bar{u}=1 \text { day }^{-1}
$$

Note that now, $\delta_{\bar{u}} \approx-0.33<0$.

Figure 5 The parameters are the following: (A.3)-(A.5),(A.8) and

$$
\bar{U}=2 \text { days } \cdot \bar{u}
$$

Acknowledgements. This work is part of the DIM LSC project ALMA on the Analysis of Acute Myeloid Leukemia. It brings together clinicians and biologists of the Inserm team 18 of UMRS 872 (Jean-Pierre Marie, Pierre Hirsh, Ruo-Ping Tang, Fanny Fava, Annabelle Ballesta, Faten Merhi) and mathematicians of the Inria teams Bang (Jean Clairambault, Annabelle Ballesta), Disco (Catherine Bonnet, José Luis Avila) and Commands (J. Frédéric Bonnans, Xavier Dupuis).

The author also thank Fabien Crauste and Thomas Lepoutre for their useful insights and advice.

\section{References}

[1] M. Adimy, F. Crauste. Mathematical model of hematopoiesis dynamics with growth factor-dependent apoptosis and proliferation regulations. Math. Comput. Modelling, 49 (11-12) 2009, 2128-2137,.

[2] M. Adimy, F. Crauste, A. El Abdllaoui. Discrete maturity-structured model of cell differentiation with applications to acute myelogenous leukemia. J. Biol. Systems, 16(3) 2008, 395-424

[3] J. L. Avila, C. Bonnet, J. Clairambault, H. Ozbay, S.-I. Niculescu, F. Merhi, R. Tang, J.-P. Marie. A new model of cell dynamics in Acute Myeloid Leukemia involving distributed delays. In 10th IFAC Workshop on Time Delay Systems, , Boston, USA, 2012, 55-60.

[4] A. Ballesta, F. Mehri, X. Dupuis, C. Bonnet, J.F. Bonnans, R. Tang, F. Fava, P. Hirsch, J.-P. Marie, J. Clairambault. In vitro dynamics of LAM patient blood sample cells and their therapeutic control by aracytine and an Flt3 inhibitor. In preparation.

[5] C. Basdevant, J. Clairambault, F. Lévi. Optimisation of time-scheduled regimen for anti-cancer drug infusion. M2AN Math. Model. Numer. Anal., 39(6) 2005, 1069-1086.

[6] F. Billy, J. Clairambault. Designing proliferating cell population models with functional targets for control by anticancer drugs. Discrete Contin. Dyn. Syst. Ser. B, 18(4) 2013, 865-889.

[7] F. Billy, J. Clairambault, O. Fercoq, S. Gaubert, T. Lepoutre, T. Ouillon, S. Saito. Synchronisation and control of proliferation in cycling cell population models with age structure. Mathematics and Computers in Simulation, 2012.

[8] J.F. Bonnans, X. Dupuis, L. Pfeiffer. Second-order necessary conditions in Pontryagin form for optimal control problems. Rapport de recherche RR-8306, INRIA, May 2013.

[9] J.F. Bonnans, P. Martinon, V. Grélard. Bocop v1.0.3: A collection of examples. Url: www.bocop.org, June 2012.

[10] G. Feichtinger, G. Tragler, V.M. Veliov. Optimality conditions for age-structured control systems. J. Math. Anal. Appl., 288(1) 2003, 47-68.

[11] P. Gabriel, S.P. Garbett, V. Quaranta, D.R. Tyson, G.F. Webb. The contribution of age structure to cell population responses to targeted therapeutics. J. Theoret. Biol., 311 2012, 19-27.

[12] L. Göllmann, D. Kern, H. Maurer. Optimal control problems with delays in state and control variables subject to mixed control-state constraints. Optimal Control Appl. Methods, 30(4) 2009, 341-365.

[13] T. Guinn. Reduction of delayed optimal control problems to nondelayed problems. J. Optimization Theory Appl., 18(3) 1976, 371-377.

[14] A. Halanay. Optimal controls for systems with time lag. SIAM Journal on Control, 6(2) 1968, $215-234$.

[15] P. Hinow, S. Wang, C. Arteaga, G. Webb. A mathematical model separates quantitatively the cytostatic and cytotoxic effects of a her2 tyrosine kinase inhibitor. Theoretical Biology and Medical Modelling, 4(1) 2007, 14.

[16] U. Ledzewicz, H. Maurer, H. Schättler. Optimal and suboptimal protocols for a mathematical model for tumor antiangiogenesis in combination with chemotherapy. Math. Biosci. Eng., 8(2) 2011, 307-323.

[17] U. Ledzewicz, H. Schättler. Optimal controls for a model with pharmacokinetics maximizing bone marrow in cancer chemotherapy. Math. Biosci., 206(2) 2007, 320-342.

[18] M.C. Mackey. Unified hypothesis for the origin of aplastic anemia and periodic hematopoiesis. Blood, 51(5) 1978, 941-956.

[19] C. Marquet, M. Adimy. On the stability of hematopoietic model with feedback control. C. R. Math. Acad. Sci. Paris, $350(3-4) 2012,173-176$. 
[20] P. Michel, S. Mischler, B. Perthame. General relative entropy inequality: an illustration on growth models. J. Math. Pures Appl. (9), 84(9) 2005, 1235-1260.

[21] H. Özbay, C. Bonnet, H. Benjelloun, J. Clairambault. Stability analysis of cell dynamics in leukemia. Math. Model. Nat. Phenom., 7(1) 2012, 203-234.

[22] D. Peixoto, D. Dingli, J.M. Pacheco. Modelling hematopoiesis in health and disease. Mathematical and Computer Modelling, 53(7,8) 2011, 1546 - 1557.

[23] B. Perthame. Transport equations in biology. Frontiers in Mathematics. Birkhäuser Verlag, Basel, 2007.

[24] J.M. Rowe. Why is clinical progress in acute myelogenous leukemia so slow? Best Practice \& Research Clinical Haematology, 21(1): 2008, 1-3.

[25] T. Stiehl, A. Marciniak-Czochra. Mathematical modeling of leukemogenesis and cancer stem cell dynamics. Math. Model. Nat. Phenom., 7(1) 2012, 166-202. 